\title{
The use of semiotic representations in reasoning about similar triangles in Euclidean geometry
}

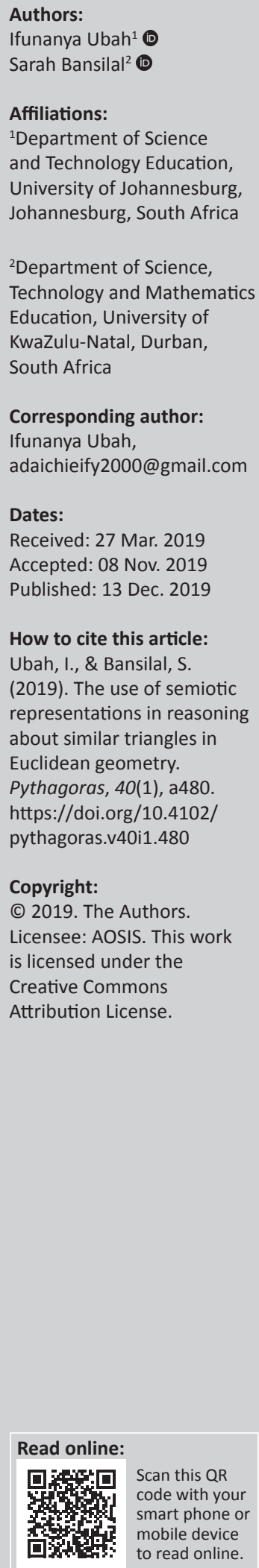

Many pre-service mathematics teachers in South Africa are apprehensive about the content of Euclidean geometry, because they did not study Euclidean geometry in high school but will be expected to teach the content when they start their teaching career. This article reports on a study that explored the role of semiotic representations in pre-service teachers' reasoning about the similarity relationship between triangles. Data were generated from the written responses of 65 pre-service mathematics teachers as well as three semi-structured interviews. Duval's notions of conversions and treatments were used as a framework to understand the pre-service teachers' struggles with negotiating movements between the visual and symbolic registers of representation. The findings revealed that many pre-service teachers struggled with identifying the similarity relationship between triangles appearing in various configurations of geometric objects. While some participants were easily able to draw upon the two registers to express the relationships, one student who initially made many errors was only able to discern the necessary relationships with the help of a concrete representation that could be physically manipulated. The study therefore provided an example of how a student's errors could be used as a learning resource to lead to meaningful learning.

Keywords: Duval; Euclidean geometry; conversion; representations; preservice mathematics teachers; similarity; similar triangles.

\section{Introduction for the study}

Mathematics outcomes in South Africa are very low and many researchers and stakeholders have expressed concerns about the poor performance in mathematics at school level, especially in geometry (Luneta, 2015; Mthembu, 2007; Singh, 2006; Van Putten, Stols \& Howie, 2010). Euclidean geometry forms part of the core curriculum of mathematics in the Further Education and Training (FET) band in South Africa; however, many learners seem to have the opinion that the study of geometry is irrelevant to their daily lives (Patkin \& Levenberg, 2012).

In South Africa, curriculum developers did not seem to be convinced about the important role of geometry in the mathematics curriculum. In 2006, as part of the many changes brought in by Curriculum 2005, for instance, the Euclidean geometry strand was made optional for those learners who opted to study mathematics in the FET band (Department of Education, 2006). One of the reasons for making it optional was the perception that teachers did not know the content well enough (Bowie, 2009). When geometry was made optional, many learners chose not to study the strand. It was found that less than $4 \%$ of the Grade 12 mathematics learners in 2008 wrote the third examination paper in mathematics where geometry was included (Van Putten et al., 2010). However, the education authorities changed their minds about the importance of geometry and in 2011 geometry was made compulsory again for these FET grades as part of the Curriculum and Assessment Policy Statements (Department of Basic Education, 2011).

When it was brought back into the core mathematics curriculum, teachers did not feel as confident about the strand since it had not been taught for such a long time. Some researchers note that teachers avoided the teaching of geometry in school because of poor mastery of Euclidean geometry (Atebe \& Shaefer, 2009; Ndlovu, 2011). Some teachers find the Euclidean geometry section difficult, even if they had studied it in high school and at tertiary level, let alone those who did not study Euclidean geometry in high school or at tertiary level. Many of our pre-service teachers were understandably anxious because they would be expected to teach the content when they start their teaching career. In order to help these students overcome their fears, the mathematics education department at a KwaZulu-Natal university designed a series of 3-hour workshops based on Euclidean geometry that were run over six weeks. One of the key Euclidean geometry concepts covered in the workshops was that of similarity of triangles, and it is this 
concept that this study focuses on. There is very little literature on students' understanding of similar triangles using a Euclidean geometry approach, and we hope that this study can add to knowledge in this field.

In this study we delve into the area of semiotics which is the study of signs and sign symbols, how these signs are used to signify actions or objects, and the interpretation of these signs (Moore-Russo \& Viglietti, 2012) As noted by Ernest (2006), a semiotic system is characterised by a set of elementary signs, a set of rules for the production and transformation of signs and an underlying meaning structure deriving from the relationship between the signs within the system (Ernest, 2006). Duval (2006) noted that mathematical objects cannot be perceived directly, but accessing them is bound to the use of representations. Hence the role of semiotic systems of representation goes beyond just a means of labelling mathematical objects but they allow a person to work on mathematical objects and with them.

The purpose of the study was to explore how semiotic representations influence students in their reasoning about the similarity relationship between triangles. To achieve this goal, the following research question was addressed:

What role do semiotic representations play in pre-service mathematics teachers' reasoning about the concept of similar triangles?

\section{Literature review}

Geometry is an essential part of mathematics and provides unique opportunities for mathematical modelling by drawing upon real-life examples (Usiskin, 2002). The study of geometry provides opportunities for learners to visualise concepts that may be related to other areas of mathematics, including trigonometry, patterns and measurement. It has links with culture, history, art and design and it is the interaction with these vital human activities that provides opportunities to make geometry lessons interesting and stimulating (Chambers, 2008). However, learners often do not view geometry as being relevant to their lives which may be because the logical and structured approach used in the study of geometry is so different from their previous experiences (Patkin \& Levenberg, 2012).

Many learners find the study of Euclidean geometry challenging (Ngirishi \& Bansilal, 2019; Singh, 2006; Van Putten et al., 2010). Nationally in 2008 only 3.8\% of the Grade 12 mathematics learners wrote the optional Paper 3 with almost half of them achieving less than $30 \%$ in that national examination paper (Van Putten et al., 2010). Furthermore, in the latest National Senior Certificate mathematics examinations in 2018, based on a random sample of Grade 12 learners' responses, it was found that, in the second mathematics paper, learners performed worst in the Euclidean geometry question based on similarity of triangles (Department of Basic Education, 2019, p. 143). This result supports Patkin and Lavenberg's (2012) contention that geometry is seen as the most complicated strand of the mathematics curriculum. It seems that the emphasis on the learning of deductive proofs of theorems, the correct use of symbolic notation and the structured requirements of providing appropriate reasons for statements that are made make the subject seem complicated (Department of Basic Education, 2019). Furthermore, the complexity of disentangling the various figures that make up more complex figural arrangements whose properties need to be discerned render the strand even more challenging for learners (Department of Basic Education, 2019).

Many students are daunted by the learning of the formal logic and deductive reasoning that are necessary elements of Euclidean geometry. One of the reasons why students find geometry difficult is the emphasis on the deductive aspect without a corresponding focus on the underlying spatial abilities (Del Grande, 1986). The use of deductive reasoning is an integral part of the study of geometry and is used to develop proofs about properties and relationships within and among figures. A proof is a set of deductive steps that are used to create a narrative starting from a known fact. It then proceeds in a step-by-step manner where each step is deduced from the result of the previous one until the unknown fact is justified (Ngirishi \& Bansilal, 2019). It is generally expected that the proof, made up of the sequential statements, should be supported by valid reasons (Serra, 1997). Mudaly and De Villiers (2004) highlight that the study of formal proof as part of the study of geometry is a useful means of developing deductive reasoning skills.

Another characteristic feature of the study of geometry is the necessary intertwining of the visual and symbolic or analytic representations where the one representation supports and underpins the others. As pointed out by Del Grande (1986), the development of spatial abilities is an important part of studying geometry. For some students it is this necessary dependence on the use of more than one representation that is experienced as a challenge. Duval (2006) argues that in geometry it is necessary to combine the use of at least two representation systems, one for verbal expressions of properties and the second one for visualisation. Duval (2006, p. 107) maintains that the 'ability to change from one representation system to another is very often the critical threshold for progress in learning'. Visualisation is the ability to 'represent transform, generalise, communicates, document, and reflect on visual information' (Hershkowitz et al., 1990, p. 75). Presmeg (1997, p. 304) focused on visualisation as a process 'involved in constructing and transforming visual images' which is the view that is taken in this study.

Bansilal and Naidoo's (2012) study focused on the use of visualisation and analytic strategies by 40 Grade 12 learners when working with problems based on transformation geometry. The findings revealed that most learners easily carried out processes and calculations in the analytic mode when responding to the tasks, showing a limited movement across the two modes which are essential for a deepening of 
understanding of geometry. The authors argued that on the one hand, as the learners' understanding improves, the learners find it easier to move across different representations. On the other hand, as learners work with different representations of an object, the different aspects emphasised by each representation contribute to a deeper understanding of the properties and relationships associated with the object (Bansilal \& Naidoo, 2012). Another study (Bansilal, 2012), which drew upon Duval's theory about transformations within and between semiotic representation systems, focused on teachers' success rates in solving problems based on the normal distribution curve. The findings of the study showed that the teachers achieved a higher success rate in processes that were based in one representational system compared to those processes that required coordination between two different representational systems.

Sinclair et al. (2016), in their review of research on geometry education, noted that there has been increased attention in recent years on visuospatial reasoning mainly in the teaching and learning of geometry, although researchers refer to this focus by different terms such as visualisation and visualising, spatial reasoning, visuospatial thinking as well as visual reasoning. Sinclair et al. (2016, p. 696) note that they have in common 'the activity of imagining static or dynamic objects and acting on them (mentally rotating, stretching etc.)'. Rivera (2011) drew attention to the complexity of reasoning about visual representations. Duval (2005), cited in Sinclair et al. (2016), identified three cognitive processes as being central to learning. These three processes are visualisation, construction (using instruments) and a discursive process for communicating argumentation and proofs (Sinclair et al., 2016). Duval argued that 'attending to the properties of a geometric figure involves dimensional deconstruction'; for example, in order to 'see' the properties of a rectangle, a student needs to concentrate on the sides and angles (cited in Sinclair et al., 2016, p. 693). However, a study by Gal and Linchevski (2010) found that students had difficulty with deconstruction of figures when asked to point out shared parts (lines or angles) of triangles that have a common side. The students commonly identified intersecting lines as a shared side or the combination of two angles, with one falling in the one triangle and the other in the second triangle, as being shared or common.

Rivera (2011) noted that depending on what activity is being done, different kinds of visual representations can be generated which could be influenced by personal intuition, related to the development of a concept or process or produced as a means of solving a problem. In their study of a Brazilian classroom, David and Tomaz (2012) argued that visual representations should form a central part of structured learning activities. However, in their analysis, they found that learners held a dominant view that geometry required the learning of rules and norms and this interfered in their learning about calculating areas using drawings. It was also found that talking about and establishing common understandings about visual representations helps learners develop a conceptual understanding of geometric concepts (Steenpass \& Steinbring, 2014).

Gal and Linchevski (2010) identified that students experienced difficulties when trying to distinguish between the various configurations present in geometric diagrams. It was a challenge for students to identify those visual characteristics that were relevant to the solution of the problems and they could be side-tracked by those characteristics that were not relevant. The authors also noted that students try to deal with mental objects as if they were physical objects by trying to transform or manipulate the objects mentally. Consequently, the difficulty of problems in geometry increases as the cognitive demand of the mental transformation increases. For example, the authors found that students found it easier to identify similar triangles in figures such as Shape 1 in Figure 1 ( $\triangle A B^{\prime} C^{\prime}$ and $\triangle A B C$, where $\angle A B^{\prime} C^{\prime}$ and $\angle A B C$ are right angles) than that of Shape 2( $\triangle K L M$ and $\triangle K N L$, where $\angle K L M$ and are $\angle K N L$ right angles) because the second shape requires a greater cognitive effort for the mental transformation.

Identifying and understanding the errors that students make during the process of constructing their knowledge has occupied the attention of many researchers. However, such an enterprise is also valuable for teachers since knowledge of these errors can be used as a learning resource in their classrooms. Chauraya and Brodie (2018) argued that teachers need learning opportunities that can allow them to develop the skills of eliciting learners' thinking about the errors they made. They found that as teachers focused on understanding learner errors, the teachers also improved their own mathematics knowledge (Chauraya \& Brodie, 2018). Going beyond just an acknowledgement of correct or incorrect answers, towards actively using the incorrect answers productively as a means to enhance understanding, may need a mind shift on the part of teachers as well as researchers. In this study we offer an example of how one student's incorrect answers were used as a resource to develop meaningful learning.

\section{Theoretical background}

According to Duval (2006), there is no mathematical processing that can be performed without using a semiotic

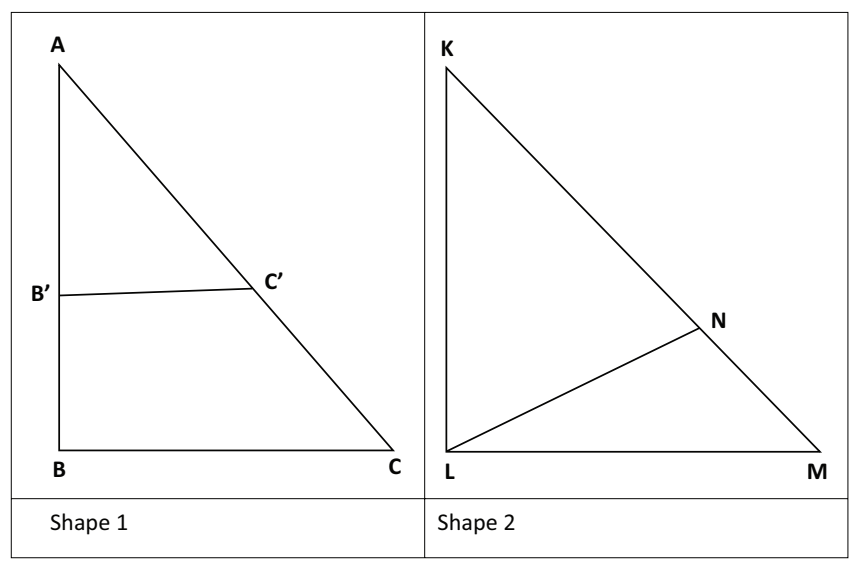

FIGURE 1: Shapes requiring different mental transformations. 
system of representation. This is because mathematical processing always involves substituting some semiotic representation for another. Duval's focus is not on single representations but on systems of representations which have rules for performing transformations of representations within the system without changing the mathematical object that is used. Duval referred to these systems of representations as registers and specified two different types of transformations of semiotic representations that can take place during any mathematical activity, namely treatments and conversions. Treatments involve transformations from one semiotic representation to another within the same system or register, while conversions involve changing the system while retaining the reference to the same objects (Bansilal \& Naidoo, 2012).

An example of a treatment could be carrying out a calculation while remaining in the same notation system. Transforming the representation $\frac{1}{4}+\frac{1}{4}+\frac{1}{4}$ into the representation $\frac{1}{2}+\frac{1}{4}$ is a treatment because the notation system has remained the same. However the transformation from $\frac{1}{2}+\frac{1}{4}$ to $0.5+0.25$ is an example of a conversion, because the algorithms for the addition of the decimal numbers are different from that of the numbers written in fractional form (Duval, 2006).

Duval (2006) notes that a conversion comes in 'for the purpose of choosing the register in which the necessary treatments can be carried out most economically or most powerfully' (p. 106). For example, when asked to show that the opposite angles of a cyclic quadrilateral are supplementary, it would be very cumbersome to write out a verbal argument, whereas it would make sense to provide a visual representation of the quadrilateral which can be used to support and clarify the argument. A further purpose of conversions, according to Duval (2006), could be to provide 'a second register to serve as a support or guide for the treatments being carried out in another register' (p. 106). An example of this function is when one is asked to solve a trigonometric equation such as: $\sin ^{2} x+\cos 2 x-\cos x=0$. The algebraic register can serve as a support to expand this trigonometric expression using the rules of algebra (once the expansion for $\cos 2 x$ is identified).

\section{Methodology}

This interpretative study was located within a six-week intervention that was designed to help pre-service mathematics teachers improve their understanding of FET Euclidean geometry. The purpose of the study was to explore how students used semiotic representations in reasoning about the similarity relationship between triangles. The participants of the study were 65 students who enrolled for the intervention. The data for the study were generated by the written responses of the participants to one task based on similar triangles, as well as semi-structured interviews that were conducted with 13 participants who volunteered to be interviewed about their understanding of the concept. The interviews were video-recorded and then transcribed verbatim by the first author.
In order to ensure reliability, the transcripts were checked by the second author against the original recordings. For the purpose of this study, we draw upon interviews with three participants: Sabelo, Celo and Vince, to highlight particular ways in which the semiotic representations were used to reason about the similarity of triangles. Sabelo and Celo had not studied geometry in the FET phase of their schooling while Vince had written the third mathematics paper in Grade 12, which was optional, and which included the study of Euclidean geometry. The purpose of the interviews was to probe their reasoning about the concepts in the written tasks. However, the interviews were also used to help improve the interviewees' understanding, hence they were interspersed with explanations of key concepts where necessary to clarify the thinking and strategies used in responding to the questions.

\section{Ethical considerations}

Ethical clearance for this research was obtained from the Research Ethics Committee of the Education Faculty at the relevant university (ethical clearance number HSS/0425/018A). After obtaining approval, each participant gave their written informed consent to participate in the research, allowing the use of their responses to the written task and interview extract for research purposes and assured anonymity in the use of these data.

\section{Results and discussions}

We discuss the participants' responses to the task which was based on identifying and naming the similar triangles that emerged from various geometric figures. The identification of the equal angles within the triangles to confirm the similarity of pairs of triangles, required knowledge of the properties of these figures which are typically studied in FET mathematics.

The item analysis for the task based on the pre-service teachers' written responses is presented in Table 1.

From the results in Table 1, $39(60.0 \%)$ out of 65 participants answered Question 2.2 correctly, while $32(49.2 \%)$ participants answered Question 2.3 correctly. Thirty (46.2\%) participants answered Question 2.1 correctly. Question 2.4, Question 2.5 and Question 2.6 recorded poor performance while Question 2.7 had the fewest correct responses (21.5\%). The difference in success rates for Question $2.2(60 \%)$ and Question $2.7(21.5 \%)$ is quite striking, considering that the underlying figure in both diagrams is that of a crossed quadrilateral. The increased difficulty of Question 2.7 supports the assertion by Gal and Linchevski (2010) that the difficulty of problems in geometry increases as the cognitive demand of

TABLE 1: Frequency of Correct, Wrong and No responses for items in the task.

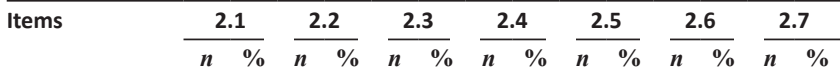

Correct response $30 \quad 46.2 \quad 3960.0 \quad 32 \quad 49.2 \quad 2030.8 \quad 1929.2 \quad 17 \quad 26.2 \quad 14 \quad 21.5$ Wrong response $\begin{array}{lllllllllllllll}28 & 43.1 & 20 & 30.8 & 23 & 35.4 & 37 & 56.9 & 35 & 53.9 & 38 & 58.5 & 42 & 64.6\end{array}$ \begin{tabular}{lllllllllllllll} 
No response & 7 & 10.8 & 6 & 09.2 & 10 & 15.4 & 8 & 12.3 & 11 & 16.9 & 10 & 15.4 & 9 & 13.9 \\
\hline
\end{tabular} 
the mental transformation increases. The mental transformation required in Question 2.7 is more difficult to achieve than that of Question 2.2 because $\triangle P Q T$ needs to be mentally reflected across a vertical line so that the new configuration of the triangle enables the identification of the matching angles in the two triangles concerned. For Question 2.2, the configuration of the triangles is such that the matching angles are easily identified. A second reason for the large number of correct responses in Question 2.2 may be because participants were very familiar with the theorem that angles on the same segment' of a circle are equal (see interview extract for Sabelo below for confirmation). The students also struggled with Question 2.4, Question 2.5 and Question 2.6 whose complex figures may have limited the participants' correct response. For questions in the task to identify the matching pairs of angles and hence the order of the triangle representation (such as $\triangle P R S$ III $\triangle P T Q$ in Question 2.2), it is helpful to move to the visual representation so that the properties of the figures could be discerned. Figure 3 presents the response of Sabelo who had problems with naming the triangles in the correct order.

In Figure 3, Sabelo has been able to identify the triangles, but he was unable to represent most of the triangles in the correct naming order. He did not have a problem with Question 2.2 however as he explained in the extract from the interview below. This shows that Sabelo was comfortable with working with the visual representation in Question 2.2 which drew upon the 'angles of the same segment are equal' theorem, and he was consequently able to represent this using the symbolic representation $\triangle P R S \| I P T Q$ :

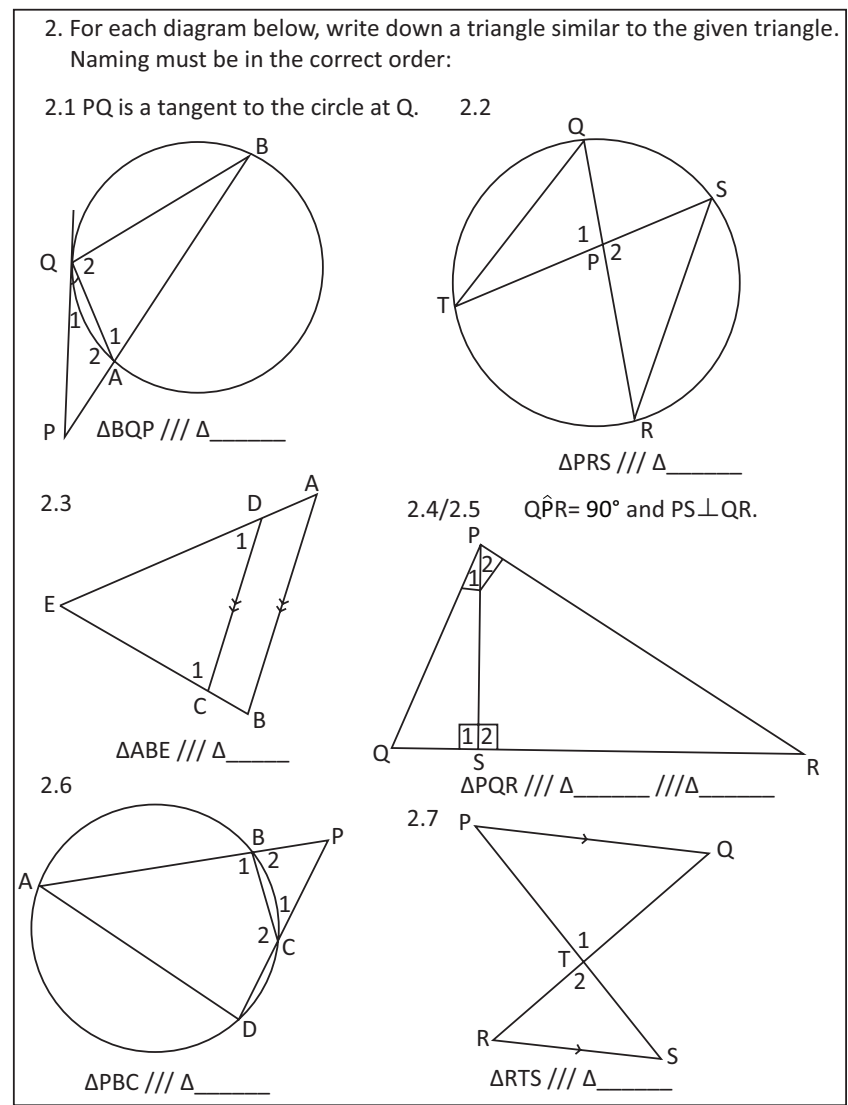

FIGURE 2: Details of the task.
R: Which of the six figures do you find very easy to solve?

Sabelo: Question 2.2.

R: Why?

Sabelo: Because the theorem involved is very easy; angles of the same segment are equal.

R: Then which angle is equal to what?

Sabelo: [Gesturing at the points $Q$ and $S$ in the figure] $\angle Q=\angle S$ [same segment], $\angle T=\angle R$, then $\mathrm{P}$ is common. Hence $\triangle P R S \| \triangle P T Q$.

As seen above, Sabelo had no problems identifying the angles that were equal in the two triangles using the 'angles in the same segment are equal' result and thereafter representing the symbolic relationship between the similar triangles correctly. The interview continued, where Sabelo was probed about other questions:

R: $\quad$ What are we required to do in Question 2.1?

Sabelo: We are required to show that $\triangle B Q P$ is similar to unknown triangle.

R: $\quad$ How do we find the unknown triangle?

Sabelo: The diagram is confusing.

R: $\quad$ Let's start by identifying the equal angles.

Sabelo: We start by identifying the equal angles; like $\angle B$ is equal to $\angle Q$ (tangent and chord theorem), $\angle Q$ in $\triangle B Q P$ is equal to $\angle P$ in another triangle $\angle P$, is equal to $\angle A$ then $\triangle B Q P$ is equal to $\triangle Q P A$

For each diagram below, write down a triangle similar to the given triangle. Naming must be in the correct order:

$P Q$ is a tangent to the circle at $Q$.

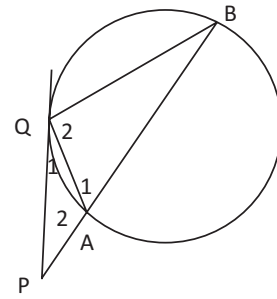

$\triangle \mathrm{BQP} / / / \triangle \underline{\mathrm{QPA}}$

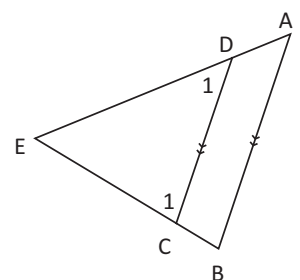

$\triangle \mathrm{ABE} / / / \triangle \underline{\mathrm{EDC}}$

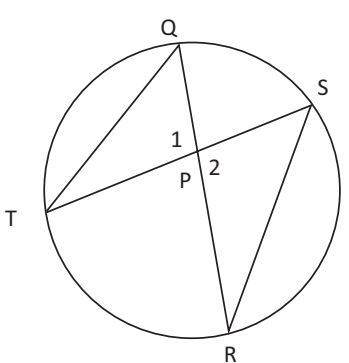

$\triangle \mathrm{PRS} / / / \triangle \mathrm{PTO}$
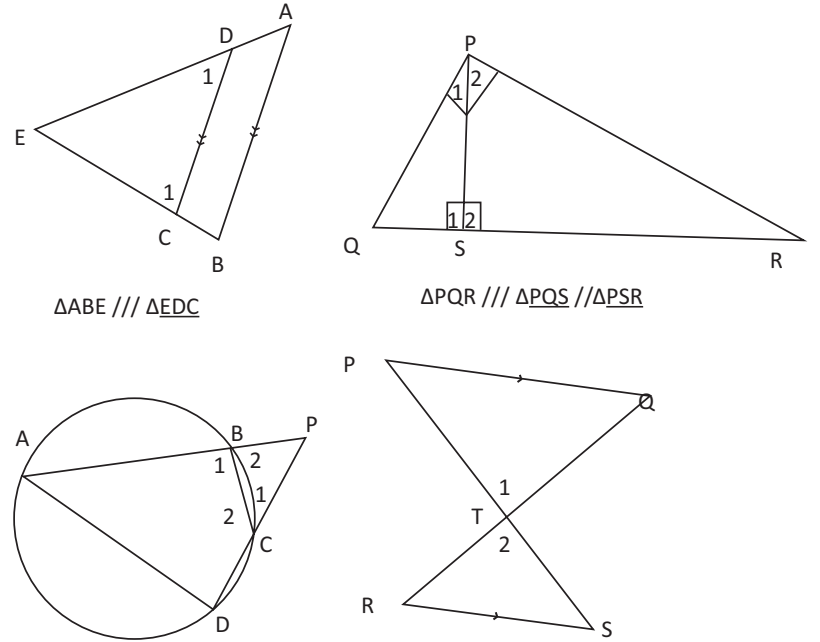

$\triangle \mathrm{PBC} / / / \Delta \underline{\mathrm{APD}}$

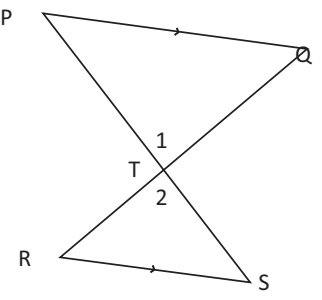

$\triangle \mathrm{RTS} / / / \Delta \underline{\mathrm{PTQ}}$

FIGURE 3: A representation of Sabelo's response. 
R:

Sabelo:

R:

Sabelo:

R:

Sabelo:
Why is $\angle Q=\angle P$ ?

Because $\angle P$ is exterior to $\angle Q$.

Yes, Then $\triangle B Q P \|{ }^{\prime} \triangle Q P A$

[Moving on to the other question] Now in Questions 2.4 and 2.5, how do we get the unknown triangles similar to $\triangle P Q R$ ? wanted to say that $\angle S$ should be common, but $S$ is not in $\triangle P Q R$. I need to study the question.

In the above extract, Sabelo's misconception that ' $\angle P$ is exterior to $\angle Q^{\prime}$ which made them equal was revealed. Based on this incorrect deduction, Sabelo has named the triangles in the order $\triangle B Q P$ and $\triangle Q P A$ respectively. Sabelo's steps can be viewed as a treatment that was carried out within the symbolic register. He has used the pairs of angles he identified as equal from the two triangles to set out the order of naming the two triangles and hence represented the similarity relationship incorrectly as $\triangle B Q P\|\|^{\prime} \triangle Q P A$. With respect to Question 2.4 and Question 2.5, he was at a loss and did not know how to figure out the triangles that were similar or identify the equal angles. Further problems with his reasoning in the visual representations are revealed in the following extract:

R: $\quad$ What of Question 2.7, how do we get the unknown triangle?

Sabelo: [He first draws the two triangles RTS and PQT separately.] $P Q$ is parallel to $R S$, so it means that $\angle Q=\angle S$ [alternate angles], also $\angle P=\angle R$, and $\angle T$ is common, hence $\triangle R T S$ II $\triangle P T Q$.

The above interview extract shows that Sabelo has once again made an incorrect deduction when working with the visual representation and identified angles incorrectly as being equal $(\angle Q=\angle S ; \angle P=\angle R)$. Furthermore, his understanding of 'common angle' is similar to the misconception identified in the study by Gal and Linchevski (2010). As he did for Question 2.1, Sabelo applied the incorrect deduction to carry out a treatment within the symbolic register to represent the similarity relationship incorrectly as $\triangle R T S$ II $\triangle P T Q$.

Vince was one of the students who correctly represented the similarity relationships for all the questions in the task and agreed to be interviewed. During the interview he was asked to explain how he arrived at the correct answer for some of the items.

$\mathrm{R}: \quad$ Looking at the given task [pointing to the script of the participant] what do you observe?

Vince: There are six different shapes included in different triangles and we are required to name triangles similar to the given triangle in correct order.

R: $\quad$ How do we find the unknown triangles? Let's take 2.3.

Vince: $\quad$ In 2.3; $\triangle A B E$, is similar to? Er let's take $\triangle D C E$. So, we have $\angle A=\angle D_{1}$ since line $D C$ is parallel to $A B$, these are corresponding, and $\angle B$ is equal to $\angle C_{1}$ also corresponding angles. Therefore, $\triangle A B E$ is similar to $\triangle D C E \ldots$
$\mathrm{R}:$

Vince:

Now in questions 2.4, 2.5, 2.6 and 2.7, how do we get the unknown triangles similar to given triangles?

[He first marked off the equal angles in the diagram.] For question 2.4 and 2.5; $\triangle P Q R$ is given; $\angle P$ [pointing to $\triangle P Q R$ ] is equal to $\angle S$ [pointing to $\triangle S P R] ; \angle Q$ [pointing to $\triangle P Q R]$ is equal to $\angle P$ from $\triangle S P R$ and $\angle R=\angle R$, hence $\triangle P Q R$ III $\triangle S P R$ [addressing the first part of question which is 2.4. Question 2.5 is the second part which required one to show that $\triangle P Q R \quad I I \triangle S Q P$ or alternately $\triangle S P R$ III $\triangle S Q P]$

From the interview extracts above, Vince is comfortable with working with the visual representation and effortlessly moves to the symbolic representation. For Question 2.2 he was able to connect the visual and symbolic representations without any hesitation, although for Question 2.4 he first spent some time working within the visual register so that he could identify the equal angles using the visual representation before expressing the relationship symbolically.

Celo's responses in Figure 4 show that he was only able to identify the pair of similar triangles, and name them in the correct order for Question 2.3, while his answers for the other six questions were wrong. Celo was interviewed about his responses.

R: $\quad$ If we look at your answer for Question 2.3, it is $\triangle A B E$ III $\triangle D C E$, how did you arrive at that answer?

For each diagram below, write down a triangle similar to the given triangle. Naming must be in the correct order:

$P Q$ is a tangent to the circle at $Q$.

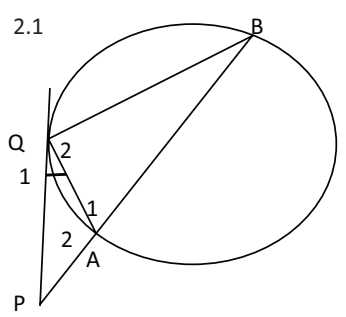

$\triangle \mathrm{BQP} / / / \triangle \underline{\mathrm{PAO}}$

2.3

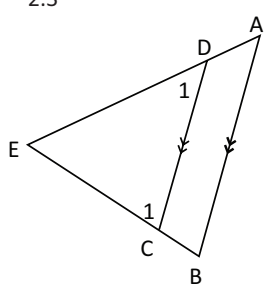

$\triangle \mathrm{ABE} / / / \triangle \underline{\mathrm{DCE}}$

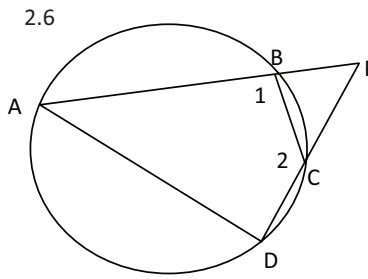

$\triangle \mathrm{PBC} / / / \triangle \mathrm{PAD}$

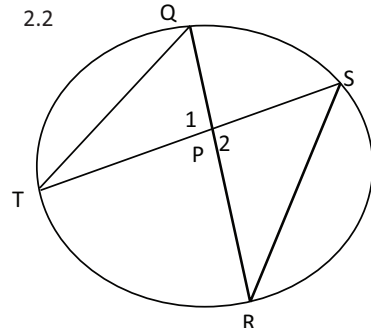

2.4/2.5 QPिR $=90^{\circ}$ and $P S \perp Q R$.

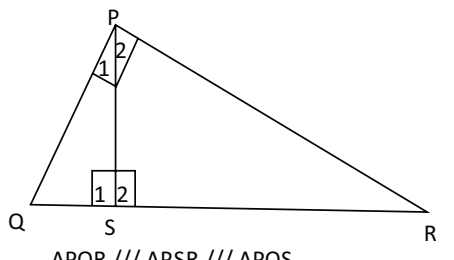

$\triangle \mathrm{PQR} / / / \triangle \underline{\mathrm{PSR}} / / / \triangle \underline{\mathrm{PQS}}$

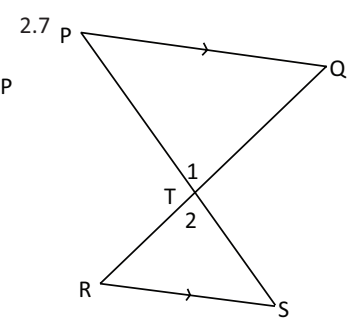

$\triangle \mathrm{RTS} / / / \Delta \underline{\mathrm{PQT}}$ $\triangle \mathrm{PRS} / / / \triangle \underline{\mathrm{QPT}}$

FIGURE 4: A representation of Celo's response. 
Celo:

This was easy because you have two parallel lines and $D C$ is cutting the big triangle and parallel to $A B$. Since the lines are parallel to each other, they divide the biggest triangle into two triangles, so the bigger triangle is similar to the smaller triangle.

His response is focused on how he identified the two triangles that are similar, but he has not mentioned the order of naming the triangles. He was then probed about this.

R:

But why would you say $\triangle D C E$, why not $\triangle C D E$ ? If you got $\triangle A B E$ similar to $\triangle C D E$ how did you work, it out so that you wrote $\triangle A B E I I I \triangle D C E$ ?

Celo: I just wrote it.

Hence, it was clear that Celo did not assign any significance to the symbolic representation $\triangle A B E\|I\| D C E$ and assumed it was sufficient to just identify the pairs of triangles that were in a similarity relationship. He seemed to have used the same reasoning for Question 2.6 - he was pleased to just identify the triangles that were similar.

The researcher spent much time explaining the significance of the order when using the III notation. The symbol III is a specialised notation, for example $\triangle A B E$ III $\triangle D C E$ shows that the two triangles are similar. However, the symbol III goes further than that and specifies the order of the corresponding angles (and sides). That is, angles $A, B$ and $E$ of $\triangle A B E$ are respectively equal to angles $D, C$ and $E$ of $\triangle D C E$, while the ratios of the lengths of the lines $A B: D C=B E: C E=A E: D E$.

In continuing, the researcher then asked him to try and work out the correct representation for the similarity of the triangles in Question 2.1. In considering $\triangle B Q P$ :
R:
So, let's try to get the angles matched for $\triangle B Q P$, then we can get the right order.
Celo: Okay er $B$ er $=Q 1$ because of tangent $\ldots P$ is the same, and $Q=A_{2}[$ mumbling $]$
$\mathrm{R}: \quad$ Right so $\triangle B Q P$ is similar to triangle?
Celo: $\quad Q A P$, but I was right [referring to his written answer that $\triangle B Q P$ II $\triangle P A Q]$ I identified the triangles, it is the right triangle.
$\mathrm{R}: \quad$ But the order was not correct.

Celo then went on to correctly represent the similarity relationship for Question 2.2. He was then probed about Question 2.4 and Question 2.5 which he found difficult to work with. The researcher explained how the order could be found by taking $\angle Q=x$, then working out $\angle P_{1}$ to be $90^{\circ}-x$, then $\angle P_{2}=x$ and $\angle R=90^{\circ}-x$. Celo understood the calculations but did not seem convinced. He said he had a different method and tried to explain it:

C: $\quad$ My method is different from yours - I use logic. I have $\triangle P Q R$ here ... now we got three triangles here. ... Now my method is like logic ... I will take this triangle [referring to $\triangle S P R$ ] and put it over here [he gestured with his hand showing that he was moving the triangle to coincide with $\triangle P Q R]$

R: $\quad$ Are you trying to move it in your head, like inverting it?

C: $\quad$ [Continues] Yes so $\mathrm{P}$ will come to this point [gesturing to point $R$ on $\triangle P Q R]$ the $S$ will move over to like this [gesturing to ' $P$ ' on $\triangle P Q R$ and 'S' on $\triangle S P R$ ].

$\mathrm{R}$ : Can you draw it for me? Can you tell me your order? C: [Celo tried to draw the triangles that were manipulated to show the corresponding pairs of equal angles but did not quite succeed. He wrote $\triangle P Q R$ III $\triangle S R P]$.

It was evident that Celo was trying to visualise how the triangle could be moved around so the two triangles were oriented so that the similarity of the shapes could be easily discerned. However, it was difficult to mentally transform the image in his mind to match the symbolic representation that was needed. At this stage, the time was up so it was arranged that Celo would meet up again and demonstrate his method. At the next meeting, Celo came prepared with three different coloured triangles that were arranged as shown in Figure 5.

In Figure 5 on the left-hand side, Celo placed the model of $\triangle P Q R$ using white cardboard on the desk with the angles marked as given. The right-hand side of Figure 5 shows two other triangles overlaid on $\triangle P Q R$. The triangles are in two other colours with $\triangle P Q S$ being dark grey while $\triangle P S R$ is made up of light grey cardboard.

Thereafter, Celo then reflected the light grey triangle across the line $S R$ and then moved the triangle so that the angle $S$ in the light grey triangle coincided with angle $P$ in the original white triangle $\triangle P Q R$ as shown in Figure 6. It could now be seen clearly that the light grey triangle was a dilation of the white triangle.

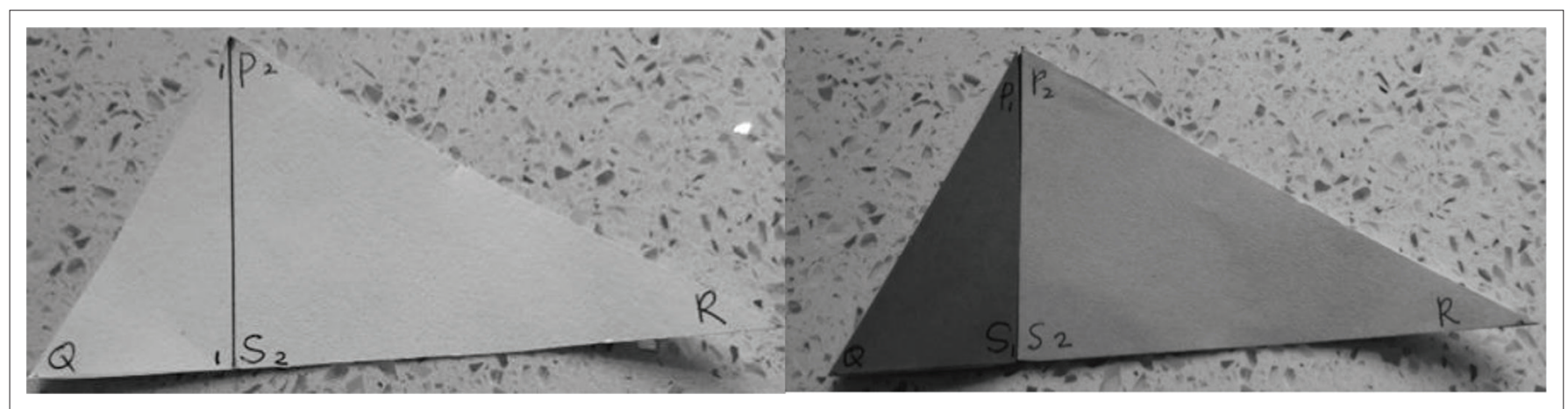

FIGURE 5: Celo's cardboard models of the triangles. 
By physically manipulating the light grey triangle, he was therefore able to easily match the pairs of equal angles corresponding to one another in the two triangles. He then proclaimed, 'Now you can see that $\triangle P Q R$ is similar to $\triangle S P R$ '. The reorientation using the physical representation helped him to identify the matching vertices which was needed for the symbolic representation. Once the order of the corresponding vertices of the triangles has been established, it becomes easier to work within the symbolic register and express the ratios of the corresponding sides that were in proportion: $P Q / S P=Q R / P R=P R / S R$. At this stage the student did not need the visualisation of the two triangles but can make the necessary deductions using just the symbolic expression, $\triangle P Q R$ III $\triangle S P R$.

Figure 7 shows how the three triangles in the original figure are rearranged to illustrate their similarity property, making it easy to recognise that $\triangle S Q P\|\| S P R \| \triangle P Q R$.

This demonstration provided insight into Celo's reasoning because it showed that Celo was dependent on the cardboard cut-outs as a physical representation that could be manipulated. He needed to 'see' the orientation of the triangles so that he could draw out the symbolic representation. This means that he could not work within the symbolic register only - that is, to carry out a treatment, using the equality relationship between the angles of the triangles to express it using the similarity notation. However, when he had the physical triangle models that could be manipulated then he was able to use the similarity notation to express the relationship between the triangles. His problem is that he needed the visualisation processes to be merged with the symbolic representation. Working within the symbolic register requires one to be convinced that if angles $S, Q$ and $P$ of $\triangle S Q P$ are respectively equal to angles $S, P$ and $R$ of $\triangle S P R$, then this means that $\triangle S P R \quad$ III $\triangle S Q P$.

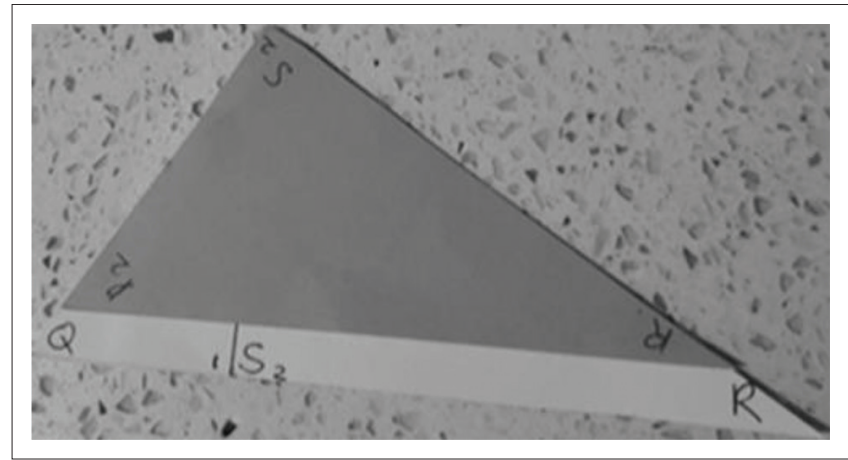

FIGURE 6: Reorientation of the light grey and white triangles.

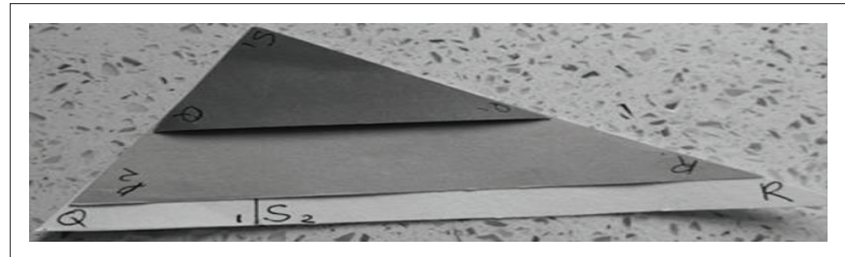

FIGURE 7: Rearrangement of the three triangles.
Celo's experiences show how important it is for students to be able to make connections between different representational registers. Duval highlighted that it is sometimes necessary to move to another register, that is, to carry out a conversion, so certain properties of an object can be discerned. Although an object in one register is the same as the object in another register, each register conveys certain properties that may not be so easily discernable in the other register. This function of conversions was illustrated by Celo's use of the concrete manipulatives which enabled him to manipulate the triangle models so that he could 'see' that one was an enlargement of the other. He was not able to discern the equal angles based on the visual representation only and needed to perform the transformations on the physical representation so that the matching angles could be identified. The interview with Celo revealed that he found the mental transformation required in the visual representation too difficult. By drawing upon his cardboard cut-outs (physical representation) he was able to carry out a physical manipulation on the triangle models. Furthermore, he was not able to engage properly with the symbolic notation of III because he used it as a sign indicating that two shapes are similar without giving any consideration to the order of the naming. The symbolic register did not give Celo access to the objects and he needed to use the manipulatives comprising a physical or concreterepresentation so that he could work out the properties of the objects.

It was clear that Celo needs more opportunities for working across the three registers of representation for the concept of similarity. However, it was to Celo's credit that he recognised that the physical representation would help him access the properties of the objects unlike Sabelo, for example, who was stuck when faced with Question 2.4 and Question 2.5 and did not have the means to move beyond this barrier. Sabelo's problem was that he did not understand the properties of the geometric figures well enough and needs more opportunities to improve his skills in this area. Clearly the understanding of geometry requires fluency in moving between the visual representation using geometric figures and the symbolic representations which make use of symbolic notations for congruency, similarity, etc. Sabelo was able to work with the symbolic register and carried out the treatments within the symbolic register but expressed the similarity relationship incorrectly because of his incorrect deductions.

\section{Conclusion}

In this article, we studied the responses of 65 pre-service mathematics teachers to a Euclidean geometry task based on similar triangles and focused on the role played by semiotic representations in identifying and naming similar triangles which arose in various configurations of geometric objects. It was found that most students struggled with the symbolic specialised similarity notation (III). The symbol III is a specialised notation that denotes which two triangles are in a similarity relationship, for example $\triangle A B E$ III $\triangle D C E$ shows that the two triangles are similar. However, the symbol III goes further than the identification and specifies the order that the vertices must be arranged when the triangles are 
configured so that the matching of the corresponding angles and sides is evident. Some students handled this challenge by carrying out mental transformations on the objects (Gal \& Linchevski, 2010). When the cognitive demand of the mental transformation was higher, students found it more difficult to identify the similarity relationship. For example, it was found that for a pair of items (Question 2.2 and Question 2.7) that were similar in configuration except that the mental transformation required for Question 2.2 was simpler than that required for Question 2.7, students performed much better on the first item.

Sometimes students can carry out treatments that are based on incorrect deductions which leads to incorrect results as in the case of Sabelo. His use of treatments in the symbolic register led to incorrect results because he incorrectly identified angles as being equal. His knowledge of the relationships and properties of the underlying geometry concepts was weak, so his incorrect deductions led him to incorrect formulations of the similarity relationships between triangles.

Duval (2006, p. 107) asserted that conversion-type activities play a crucial role in developing understanding of a concept and it is often the case that 'the ability to change from one representation system to another is very often the critical threshold for progress in learning' and the activity of conversion can lead to the mechanisms underlying understanding. This statement is true especially for the study of Euclidean geometry where the artefacts are visual representations of configurations of objects such as angles, lines, polygons, circles, etc. In this study we found that skilful conversion movements between the visual and symbolic registers led to success in solving Euclidean geometry problems as illustrated by Vince's approach. Duval further notes that a key purpose of conversions in the learning process is to provide 'a second register to serve as a support or guide for the treatments being carried out in another register' (Duval, 2006, p. 106). Vince was able to use the symbolic register skilfully while the visual representation served as a support from which he derived the necessary symbolic representations. Vince used the visual representation minimally to identify the matching angles using properties that were discerned from the visual representation and moved easily to express the similarity relationships based on these properties.

For one student, the symbolic and the visual representation did not provide him enough access to the objects to allow him to discern the relationship and it was only after carrying out the rigid transformations using concrete representations of the triangles that he was he convinced about the similarity relationship and the order of the naming. Celo needed the comfort of the physical representation of the objects that can be manipulated or rigidly transformed in order to facilitate the visual representation showing that the one triangle is an enlargement or dilation of the other as shown in Figure 7, Figure 8 and Figure 9. This rearrangement allowed Celo to 'see' the relationships between the objects in the visual representation and he could then express these relationships using the symbolic representation.

A salient point relates to the fact that Vince had elected to study Euclidean geometry in school although it was not compulsory while Sabelo and Celo did not have that opportunity. The geometry workshop was designed to help students such as Celo and Sabelo. The results of this study showed that the students need much more help in navigating these concepts forming part of the Euclidean geometry curriculum. Celo's initiative in making the cardboard cutouts helped him to concretise some of the relationships embodied in the similarity of triangles concept. Perhaps such teaching aids may be useful for other students such as Sabelo who did not seem to see the connections between equiangular triangles and enlargements or reductions of the triangles, which are key to the concept of similarity. Much of the earlier work in school in the earlier grades that focus on rigid transformations and enlargements or reductions of figures are meant to form the basis for this later work on similarity. Hence, the use of these concrete manipulations is necessary for students to develop a more robust understanding of the concept. This suggestion resonates with the advice given by Zazkis et al. (1996, p. 455) that 'moving across [considering equivalent representations] in order to move up [increasing abstractions], at a rate appropriate for [the learners' needs], may help them to make the connections necessary'. Although moving across by deliberately drawing upon other representations may not be easy, Zazkis et al. recommend such actions so that the students are able to develop a 'a richer and more useful understanding of complex ideas'.

In conclusion, we hope that this contributes some new knowledge in the field in terms of how learners' misconceptions or errors could be turned into a resource to promote meaningful learning (Chauraya \& Brodie, 2018), as was done in the case of Celo. We consider that such a mind shift among researchers is important so that participants derive direct benefit from the research process, instead of the situation where participants' errors are identified, elaborated and explained in a report, but very little feedback is given to the learners about their wrong answers.

\section{Acknowledgements}

We acknowledge Mdutshekelwa Ndlovu of the University of Johannesburg for proofreading the manuscript before submission, as well as Moses Mogambery who designed the activities and conducted the workshops.

\section{Competing interests}

The authors declare that they have no financial or personal relationships that might have inappropriately influenced the writing this article.

\section{Authors' contributions}

I.U. was responsible for the initial analysis and conducting of interviews as well as drawing up the first draft. S.B. refined the analysis as well as the article. 


\section{Funding information}

This research was funded as part of the postdoctoral research support provided by University of KwaZulu-Natal.

\section{Data availability statement}

Data sharing is not applicable to this article.

\section{Disclaimer}

The views and opinions expressed in this article are those of the authors and do not necessarily reflect the official policy or position of any affiliated agency of the authors.

\section{References}

Atebe, H.U., \& Schäfer, M. (2009). The face of geometry instruction and learning opportunities in selected Nigerian and South African high schools. In M. Schäfer \& C. McNamara (Eds.), Proceedings of the 17th Annual Conference of the Southern African Association for Research in Mathematics, Science and Technology Education (pp. 7-21). Grahamstown: SAARMSTE.

Bansilal, S. (2012). Using conversions and treatments to understand students' engagement with problems based on the normal distribution curve. Pythagoras, 33(1), 37-49. Art. \#132, 13 pages. https://doi.org/10.4102/pythagoras. v33i1.132

Bansilal, S., \& Naidoo, J. (2012). Learners engaging with transformation geometry. South African Journal of Education, 32(1), 26-39. https://doi.org/10.15700/saje. v32n1a452

Bowie, L. (2009, June). What is mathematics paper 3 for? Marang Centre for Mathematics and Science Education. Marang News, Issue 5.

Chauraya, M., \& Brodie, K. (2018). Conversations in a professional learning community: An analysis of teacher learning opportunities in mathematics. Pythagoras, 39(1), 1-9. https://doi.org/10.4102/pythagoras.v39i1.363

Chambers, P. (2008). Teaching mathematics. London: Sage.

David, M., \& Tomaz, V. (2012). The role of visual representations for structuring classroom mathematical activity. Educational Studies in Mathematics, 80(3) 413-431. https://doi.org/10.1007/s10649-011-9358-6

Del Grande, J.J. (1986). Can grade two children' spatial perception be improved by inserting a transformation geometry component into their mathematics programs? Dissertation Abstracts International, 47(1), 3689A.

Department of Basic Education. (2011). National senior certificate 2018. Diagnostic report. Pretoria: DBE.

Department of Basic Education. (2019). National senior certificate 2018. Diagnostic report. Pretoria: DBE.

Department of Education. (2006). The national policy framework for teacher education and development in South Africa. 'More teachers; Better teachers'. Pretoria: Department of Education.

Duval, R. (2006). A cognitive analysis of problems of comprehension in the learning of mathematics. Educational Studies in Mathematics, 61(1-2), 103-131. https://doi. $\mathrm{org} / 10.1007 / \mathrm{s} 10649-006-0400-z$
Ernest, P. (2006). A semiotic perspective of mathematical activity: The case of number Educational Studies in Mathematics, 61(1-2), 67-101. https://doi.org/10.1007/ s10649-006-6423-7

Gal, H., \& Linchevski, L. (2010). To see or not to see: analyzing difficulties in geometry from the perspective of visual perception. Educational Studies in Mathematics, 74(2), 163-183. https://doi.org/10.1007/s10649-010-9232-y

Hershkowitz, R., Ben-Chaim, D., Hoyles, C., Lappan, G., Mitchelmore, M., \& Vinner, S (1990). Psychological aspects of learning geometry. In P. Nesher \& J. Kilpatrick (Eds.), Mathematics and cognition: A research synthesis by the International Group for the Psychology of Mathematics Education (pp. 70-95). Cambridge: Cambridge University Press.

Luneta, K. (2015). Foundation phase teachers' knowledge of geometry. South African Journal of Childhood Education, 4(3), 71-86. https://doi.org/10.4102/sajce.v4i3.228

Moore-Russo, D., \& Viglietti, J.M. (2012). Using the K5 Connected Cognition Diagram to analyze teachers' communication and understanding of regions in threedimensional space. The Journal of Mathematical Behavior, 31(2), 235-251. https://doi.org/10.1016/j.jmathb.2011.12.001

Mthembu, S.G. (2007). Instructional approaches in teaching Euclidean Geometry in Grade 11. Unpublished master's thesis. Durban: University of KwaZulu-Natal.

Mudaly, V., \& De Villiers, M. (2004, July). Mathematical modelling and proof. Paper presented at the 10th Annual National Congress of the Association for Mathematics Education of South Africa, University of the North West, Potchefstroom

Ndlovu, M.C. (2011). University-school partnerships for social justice in mathematics and science education: The case of the SMILES project at IMSTUS. South African Journal of Education, 31(3), 419-433. https://doi.org/10.15700/saje.v31n3a538

Ngirishi, H., \& Bansilal, S. (2019). An exploration of high school learners' understanding of geometric concepts. Problems of Education in the 21st Century, 77(1), 82-96. https://doi.org/10.33225/pec/19.77.82

Patkin, D., \& Levenberg, I. (2012). Geometry from the world around us. Learning and Teaching Mathematics, 13(1), 14-18. Retrieved from http://www.amesa.org.za/ Patkin.pdf

Presmeg, N.C. (1997). Generalization using imagery in mathematics. In L.D. English (Ed.), Mathematical reasoning: Analogies, metaphors and images (pp. 299-312). Mahwah, NJ: Lawrence Erlbaum Associates.

Rivera, F. (2011). Towards a visually-oriented school mathematics classrooms: Research, theory, practice, and issues. New York: Springer.

Serra, M. (1997). Discovering geometry: An inductive approach (2nd ed.). Berkeley, CA: Key Curriculum Press.

Sinclair, N., Bussi, M.G.B., De Villiers, M., Jones, K., Kortenkamp, U., Leung, A., \& Owens, K. (2016). Recent research on geometry education: An ICME-13 survey team report. ZDM, The International Journal on Mathematics Education, 48(5), 691-719. https://doi.org/10.1007/s11858-016-0796-6

Singh, R.I. (2006). An investigation into learner understanding of the properties of selected quadrilaterals using manipulatives in a grade eight mathematics classes. Unpublished masters' thesis, University of KwaZulu-Natal, Durban, South Africa.

Steenpass, A., \& Steinbring, H. (2014). Young students' subjective interpretations of mathematical diagrams: Elements of the theoretical construct 'frame-based interpreting competence'. ZDM, The International Journal on Mathematics Education, 46(1), 3-14. https://doi.org/10.1007/s11858-013-0544-0

Usiskin, Z. (2002). Teachers need a special type of content knowledge. ENC Focus, 9(3), 14-15.

Van Putten, S., Stols, G., \& Howie, S.J. (2010). Making Euclidean geometry compulsory: Are we prepared? Perspectives in Education, 28(4), 22-31.

Zazkis, R., Dautermann, J., \& Dubinsky, E. (1996). Coordinating visual and analytical strategies. A study of students' understanding of the group D4. Journal for Research in Mathematics Education, 27, 435-457. 